\title{
Hearing the Voice of Faculty: Global Recommendations for Faculty Recognition in Higher Education Institutions
}

Agueda Benito, , a and Fionna Scott-Milligan ${ }^{b}$

aLaureate International Universities, Baltimore/Maryland, United States

bMedia Design School, Auckland, New Zealand

Submitted: December 23, 2018 | Editor-reviewed: December 24, 2018 | Accepted: December 25, 2018 |

Published: December 26, 2018

\begin{abstract}
Faculty constitutes one of the most important elements of higher education institutions. Their role is key in curriculum development, in guiding students' learning and driving their engagement, in conducting research, and in the overall success of their institutions. This paper presents the results of a number of faculty interviews and a focus group carried out in nine different Laureate institutions, operating in eight different countries. Faculty members were asked about professional recognition, its importance, and how it should occur. The results of the study show a lot of consistency, starting with the conviction of its importance, and then the differentiation between an environment of recognition and appreciation, and the need for formal recognition opportunities. Faculty shared some other interesting ideas and aspirations, providing good guidance for institutions that are interested in enhancing faculty recognition as part of their continuous improvement process.
\end{abstract}

Keywords: faculty recognition, faculty work, faculty rewards, faculty appreciation, faculty satisfaction

\section{Introduction and Background}

Faculty constitutes one of the most critical elements of higher education institutions. Their role is vital in guiding students' learning, driving their engagement, developing curriculum, conducting research, and contributing to the overall success of their institutions. In a recent study of faculty at a large university in the western United States, appreciation and recognition were found to be essential to overall faculty satisfaction, including satisfaction with an institution as a place to work (Sahl, 2017). While broader recognition programs have been established for some time in Laureate Education institutions, this paper reconsiders recognition from the perspective of current faculty in nine different Laureate institutions operating in eight different countries.

Fundamentally, all forms of recognition address a human need for appreciation. The American philosopher and psychologist William James said the deepest principle in human nature is the craving to be appreciated. Some 50 years later, the American psychologist Abraham Maslow proposed a theory of human motivation that identified the desire for respect from others as part of a hierarchy of human needs. While the impact of reward in the workplace has been the focus of numerous studies since the early 1900s, much has been written in both academic literature and business commentary of the late 20th and early 21 st century about the value of

*Author correspondence: agueda.benito@laureate.net

Suggested Citation: Benito, A. \& Scott-Milligan, F. (2018). Hearing the Voice of Faculty: Global Recommendations for Faculty Recognition in Higher Education Institutions. Higher Learning Research Communications, 8(2), 1-8. http://dx.doi.org/10.18870/hlrc.v8i2.433 
formal recognition programs and informal or social recognition in enhancing employee motivation and engagement as well as increasing productivity and reducing staff turnover.

Research suggests that when employees perceive that their work is valued, their satisfaction and productivity rise and they are motivated to maintain or improve excellent work (Roberts, 2005). In recent years, there has been a proliferation of formal recognition programs within a wide range of industries, including higher education, that supports the notion that employees will become more engaged and respond positively to appreciation, expressed through recognition of their work (Brun \& Ninon, 2008), particularly in difficult times (MacLeod \& Clarke, 2009). Mirroring recent trends in the evolution of management practices that have seen positive leadership and gratitude practices in the workplace (Cameron, 2013), recognition of staff has also changed. Extrinsic rewards, external to the work itself, such as pay raises, bonuses, and benefits reflect a time when work was generally more routine and bureaucratic. Researcher and business consultant Kenneth Thomas suggests that while these are still central to employment, they are now less important than the intrinsic rewards that employees get from doing meaningful work and performing it well. He concluded that employees in contemporary organizations with flat structures and agile, employee-led teams are required to use judgment and initiative to a much greater extent and are more strongly driven by intrinsic rewards (Thomas, 2009).

The American Psychological Association (APA) conducted an online survey in 2012 that found that North American employees "who feel valued are more likely to report better physical and mental health, as well as higher levels of engagement, satisfaction, and motivation, compared to those who do not feel valued by their employers" (American Psychological Association, 2012).

At the same time, the recognition industry was reported to be worth 46 billion dollars in North America (Bersin, 2012). Recognition is not solely a North American practice, however. In a 2012 study that considered predictors of overall job satisfaction across 48 countries in four global regions (Asia, North America, Europe, and Latin America) Andreassi, Lawter, Brockerhoff, \& Rutigliano found that recognition received from doing a good job, along with a sense of accomplishment from one's work, teamwork, and ability to balance one's work and personal life were significant predictors of job satisfaction. Importantly, regardless of culture and geography, the most significant drivers of job satisfaction were found to be recognition and accomplishment. Andreassi et al. concluded that, regardless of the cultural context, employees respond positively to intrinsic and extrinsic motivators, and institutions need to address both intrinsic and extrinsic rewards to enhance employee motivation and increase job satisfaction (Andreassi et al., 2012).

In addition to formal recognition programs, current recognition theories suggest that recognition should be authentic, informal, personalized/individualized, frequent, aligned with the mission of the employing institution, and not just top to bottom but also peer to peer (Bersin, 2012). A 2016 analysis by Gallup found that only one in three U.S. workers strongly agreed they received recognition or praise for doing good work in the previous week. The analysis concluded that recognition should be given frequently and in a timely way to those who deserve it so that employees value being recognized by their managers for performing a job well (Mann \& Dvorak, 2016; McDonald, n.d.).

As noted above, Sahl (2017) concluded that appreciation and recognition are important to overall faculty satisfaction and, ultimately, their satisfaction with the institution as a place to work. Reconsideration of faculty recognition from the perspective of current Laureate faculty, gathered through a number of interviews and a focus group, concludes that universities must take faculty recognition seriously through timely, formal and informal acknowledgment of a faculty member's or faculty team's behavior that has clearly gone beyond normal expectations and supports the 
institution's goals and values. This requires utilizing both a top-down approach that requires leadership to implement formal recognition plans and offer training, such as that offered by Manchester Metropolitan University, U.K., to all managers; and a bottom-up approach that asks $\mathrm{HR}$ and academic leaders to work in collaboration to maximize opportunities to create an institutional environment of appreciation and recognition. Asking how faculty would like to be recognized, and how appreciation can be shown, is a vital step toward making sure that recognition efforts will be appropriate

\section{Description of the Study}

The present study is of a qualitative nature, consisting of two phases. Phase one, six semi structured interviews; and phase two, one focus group.

During September 2018, six faculty members of different ages and backgrounds (Health Sciences, Business, Design, Economics, and Mathematics) were identified. All of them taught in various degree programs at the following Laureate institutions: Torrens University Australia (Australia); Stamford International University (Thailand); UPES (India); Universidad Europea de Madrid (Spain); Universidad Peruana del Norte (Peru), and Pearl Academy (India).

After learning the goal of the study, these faculty agreed to participate in individual semistructured interviews (phase one of the study), where the following guiding questions were presented to them in advance:

- What would you consider the most relevant achievements faculty could reach?

- How do you think faculty would like to be recognized?

- By whom? In front of whom?

- How important are awards and rewards for faculty?

- Would you agree that more recognition brings along better performance? Why?

- If you had to design a faculty recognition plan, what would it look like?

All the initial interviews took place according to the same protocol guide. They were recorded (and in some cases they were additionally answered in writing), and their thematic content was analyzed. The first draft report was then produced and constituted the discussion material for the focus group (phase two of the study).

Another three faculty members participated in the focus groups. They also had different backgrounds (Design, Human Resources, and Physics) and worked in another three Laureate institutions: Media Design School (New Zealand), INTI (Malaysia), and Istanbul Bilgi University (Turkey).

\section{Some Prior Ideas}

During the multiple conversations with faculty, some interesting ideas came up more than once. The authors of this paper identified the following as relevant thoughts that other faculty and academic leaders might appreciate reflecting on:

- Giving back is in the nature of faculty members, but recognition is very important for them too.

- Recognition and incentives do not transform a bad instructor into a good one, but it can help the good ones become even better.

- Professors are knowledge workers and intrinsic motivation is key.

- More than recognition itself, faculty appreciate that their institution cares for them and values their work. 
- Often obtaining the students' recognition matters as much as, or even more than, recognition from managers.

- Recognition of teaching should be the first concern, followed by research/industry connections.

- Junior and senior faculty appreciate different forms of recognition. Juniors need more explicit recognition from managers.

- Small achievements and reaching relatively modest milestones also matter. Recognition should not be limited to one professor of the year.

- The best part about recognition is feeling you can inspire other faculty with the things you do. Sharing of best practices and even mentoring should follow recognition.

- Good faculty might not be good sales people and may not always share their achievements effectively.

- Transparency, objectivity, and measures are recurrent concepts when talking about recognition.

- Flexibility, time, and having their voices heard are common aspirations for faculty. Time in particular is highly appreciated as a reward.

- Team recognitions help create a more profound sense of belonging and should be enhanced.

- Cultural adaptations need to be made, so that rewards are meaningful and valuable for each group of individuals.

\section{Faculty Aspirations}

When talking to faculty about recognition, one very interesting finding was that the participants had some prior requests to share before addressing recognition itself. They mentioned two necessary conditions so that any recognition program could be built on a solid base.

First, they referred to a work environment that is well organized and transparent, where responsibilities and expectations are clear. This seems to be a very basic element that could be considered a hygienic factor that reduces demotivation and disengagement of faculty.

Second, they mentioned the importance of individualization-the appreciation of the uniqueness of individuals. Since each faculty member is different, individual strengths should be identified and celebrated and tasks and projects that get assigned should be aligned with individual interests and strengths.

After these two comments faculty addressed recognition-what they would like to be recognized for and how.

Many of their comments referred to the importance of an environment of recognition and appreciation, where immediate, informal recognition takes place, and where positive communication is a common practice. They said it is ok to recognize the "big things," but recognizing the smaller things that they achieve on a daily basis was found even more relevant.

Additionally, faculty felt that the opportunities for formal recognition were also key. They defended the importance of the recognition mechanisms being inclusive (why just for one if more deserved it) and transparent (so that they knew what they needed to do themselves and that they trusted the judgement of others). Students should also be key informers and recipients of information. 


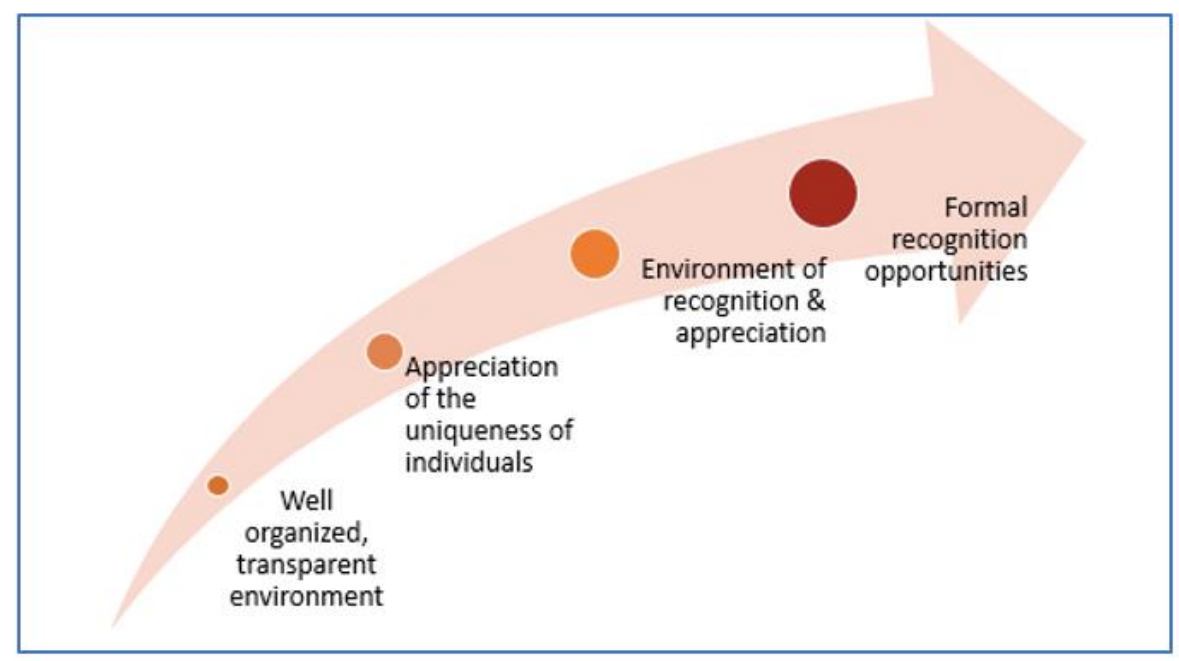

Figure 1. Faculty aspirations.

The next two sections of this paper develop more in-depth thoughts regarding the last two aspirations expressed by faculty.

\section{An Environment of Recognition and Appreciation}

When faculty described the importance of creating an environment of recognition and appreciation, they referred to the idea of flowing recognition of their achievements. Faculty also mentioned the importance of finding the right balance between individual and team recognition. They thought the following were relevant achievements, but they are often taken for granted and seldom celebrated. They thought it would be a good idea to appreciate and recognize achievements such as:

- The publication of a paper

- A presentation at a conference

- An external award

- An internal promotion

- Participation/organization of an industry event

- Participation/organization of some social responsibility or community activity

- Team achievements, like accreditations or institutional recognitions

- Student satisfaction above a certain level

- Punctuality, attendance, and commitment

It seems that faculty do not expect extraordinary celebrations of all these things, but they did mention the following mechanisms of recognition:

- Thank you emails

- Thank you bulletin board

- Thank you cafeteria vouchers

- Going out for a coffee/drink

- Small appreciation gifts 
- Positive embarrassment in departmental meetings or informal gatherings

\section{Formal Recognition Opportunities}

The interactions with all faculty left little doubt that formal recognition is also welcome and can enhance their motivation and engagement. In this case, it seems to be key that the rules of the game are clear: that recognition requirements be totally transparent, well established, and communicated to all faculty members. Faculty mentioned two very interesting mechanisms of formal recognition: newsletters and awards.

Newsletters are found to be an effective mechanism to provide the broader community with a regular update regarding faculty achievements. Videos of the interviews with the protagonists were considered more effective ways of recognition than written interviews, articles, or reports. They recommended that newsletters be distributed via email and linked to social media channels. For faculty, it was key that communication to students was ensured. Newsletters can have pre-established sections and constitute a very interesting way to highlight the institutional priorities and values, and they can provide an effective window for the recognition of teaching, research, industry connections, etc. They can showcase collaboration, internationality, and social responsibility and capture and broadcast internally any external award or recognition.

Awards are also an expectation for faculty members. They are aspirational and not present in their regular activity, but are still appreciated. Faculty described their number one priority as teaching and mentoring their students, and this is the first category of awards they recommend. Scholarship and industry connections were mentioned immediately after. As described before, faculty insisted on the importance of establishing transparent and measurable requirements and protocols, as well as the inclusion of external voices, in particular those from students and industry. Additionally, faculty questioned the restrictedness of traditional awards, where often one faculty member is recognized despite there being many others who might meet the criteria. preferences:

When discussing rewards, the faculty members identified the following as their

- Time to work on what faculty enjoy the most

- Flexibility to distribute tasks and intensity according to faculty needs

- Funds to do research

- Opportunities for development, in particular attendance at and participation in conferences, as well as having the opportunity to have international experiences in other institutions for a given period of time.

- Eventually, monetary incentives

The existence of formal promotions and career advancement opportunities needs to be part of the working conditions for faculty members. Beyond their teaching duties, faculty are generally expected to take over certain managerial roles, assume certain administrative tasks, and participate in professional development activities. Additionally, a significant number of faculty members carry out research activities, and they also contribute to various other institutional initiatives, aiming to serve the local, national, and international communities, promote the university, and support students in extracurricular activities, etc. During the interviews, faculty expressed that they would like to see clear alignment between the actual dedication of faculty, according to the institutional needs, and the opportunities for them to be promoted. Faculty also mentioned the importance of linking promotion to recognition, and recognition to promotion. In 
their annual performance review, they thought that the recognitions attained during the period considered should have a substantial weight.

\section{Recommendations}

After hearing the voice of faculty, the authors of this paper can conclude that faculty recognition should be a key component of the institutional strategy, possibly one of the investments with the most impactful, positive return.

Recognition must be multidimensional and transparent, formal and informal, and come from numerous stakeholders, including students; sometimes it only needs to bring appreciation; and other times it must provide the right rewards.

We hope that higher education institutions will understand the importance of taking faculty recognition very seriously. It is our opinion that it would be the responsibility of the top leadership to define and implement formal recognition plans. Additionally, middle managers, like heads of departments, should receive training regarding faculty recognition so that they play a more positive role in the engagement of their teams. It is also our strong belief that human resources teams and academic leaders should work in collaboration to maximize the opportunities to create an institutional environment of appreciation and recognition.

\section{References}

American Psychological Association. (2012, March 8). APA survey finds feeling valued at work linked to well-being and performance. [Press release]. Retrieved from https://www.apa.org/news/press/releases/2012/03/well-being.aspx

Andreassi, J., Lawter L., Brockerhoff, M., \& Rutigliano, P. (2012). Job satisfaction determinants: A study across 48 nations. In Tang, J. (Ed). Proceedings of 2012 Annual Meeting of the Academy of International Business-US North East Chapter: Business Without Borders. Fairfield CT: Sacred Heart University.

Bersin, J. (2012, June 13). New research unlocks the secret of employee recognition. [Blog post]. Retrieved from https://www.forbes.com/sites/joshbersin/\#19f2bfac3501

Brun, J. P., \& Dugas, N. (2008). An analysis of employee recognition: Perspectives on human resources practices. The International Journal of Human Resource Management, 19, 716-730.

Cameron, K. S. (2013). Practicing positive leadership: Tools and techniques that create extraordinary results. San Francisco: Berret-Koehler.

MacLeod, D., \& Clarke, N. (2009). Engaging for success: Enhancing performance through employee engagement. Retrieved from https://dera.ioe.ac.uk/1810/1/file52215.pdf

Mann, A., \& Dvorak, N. (2016, June). Employee recognition: Low cost, high impact. [blog post] Retrieved from https://www.gallup.com/workplace/236441/employee-recognition-low-cost-highimpact.aspx?g source=RECOGNITION\&g medium=topic\&g campaign=tiles

McDonald, M. (n.d.). Do your measures make employees mad? Or motivate them? [blog post] Retrieved from http://www.gallup.com/workplace/231659/performance-measures-motivate-maddenemployees.aspx?g source=EMPLOYEE ENGAGEMENT\&g medium=topic\&g campaign=tiles 
Manchester Metropolitan University, UK, Department of Human Resources. (n.d.). Recognising, rewarding and engaging your team: An introduction. Retrieved from https://www2.mmu.ac.uk/media/mmuacuk/content/documents/human-resources/a-z/guidanceprocedures-and-handbooks/Recognising Rewarding and Engaging your Team.pdf

Roberts R. L. (2005). The relationship between rewards, recognition and motivation at an insurance company in the Western Cape. [Masters thesis]. University of The Western Cape. Available from Core. core.ac.uk

Sahl, A. (2017). The importance of faculty appreciation and recognition: A case study of one institution. Humboldt Journal of Social Relations, 1,(39), 246-259.

Thomas, K. W. (2009, November-December). The four intrinsic rewards that drive employee engagement. Ivey Business Journal: Improving the Practice of Management. https://iveybusinessjournal.com/publication/the-four-intrinsic-rewards-that-drive-employeeengagement/

\section{Acknowledgements}

- $\quad$ Katie Canteri, Torrens University Australia

- $\quad$ Serkant Cetin, Istanbul Bilgi University

- $\quad$ Piyada Dasri, Stamford International University

- $\quad$ Alka Dwivedi, UPES

- Jesús Muñoz, Universidad Europea de Madrid

- Jim Murray, Media Design School

- $\quad$ Kannaki S. Narayansany, INTI

- Hernán Vargas, Universidad Privada del Norte

- Rupali Yadav, Pearl Academy 